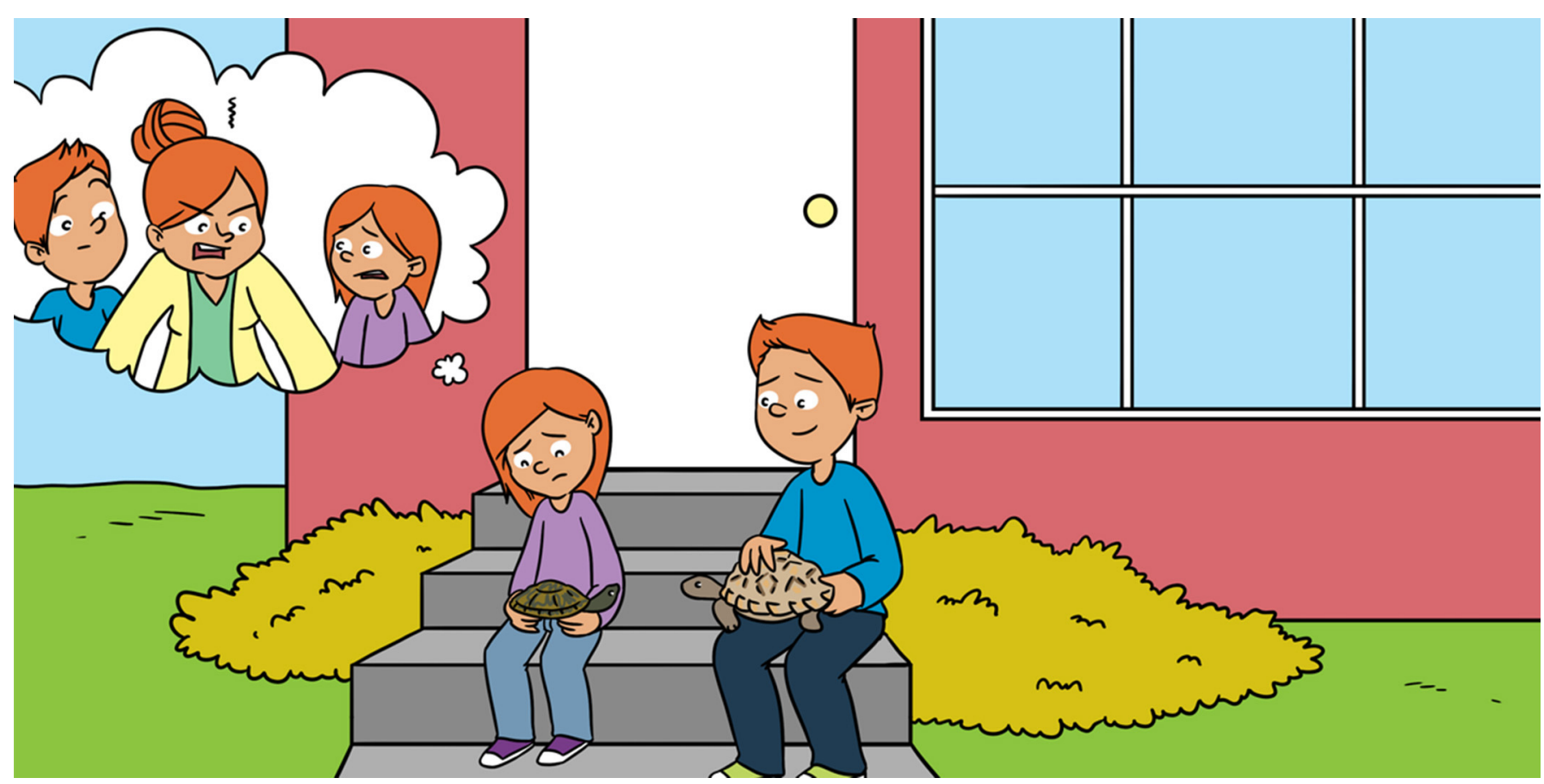

\title{
WHY ARE SOME KIDS MORE SENSITIVE TO THEIR ENVIRONMENTS?
}

\section{Margarett McBride ${ }^{1^{*}}$ and Eva H. Telzer ${ }^{2}$}

${ }^{1}$ Strengths, Assets, and Resilience Lab, Department of Psychology and Neuroscience, University of North Carolina at Chapel Hill, Chapel Hill, NC, United States

${ }^{2}$ Developmental Social Neuroscience Lab, Department of Psychology and Neuroscience, University of North Carolina at Chapel Hill, Chapel Hill, NC, United States

YOUNG REVIEWER:

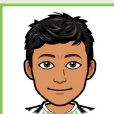

LUCAS

AGE: 15
Do you know the difference between a turtle and tortoise? They look a lot alike, but where they are suited to live effects their lives in different ways. This is true for children as well. Two children who grow up in the same house can have different life outcomes, too. Why is this? It turns out that some children are really sensitive to their environments. Sensitive children benefit the most when they are in positive environments, like those in which they have close relationships with their parents; but they suffer the most in negative environments, like those with a lot of conflict. Other children are resilient to their environments and do well in most environments. These differences in sensitivity are described by a hypothesis called the differential susceptibility model. In this article, we will explain how scientists can look at the brain to identify sensitive and resilient teens. 


\section{DEPRESSION}

A mental health disorder that impairs life by impacting mood and causing a loss of interest in activities.

\section{DIFFERENTIAL} SUSCEPTIBILITY

A hypothesis used to explain why people have varying levels of outcomes in response to their environment.
Many changes happen during the adolescent years (13-19), like puberty, changes in social relationships, and brain development. Around this time, teenagers begin to hang out with their friends more and they generally care more about social acceptance. While some teenagers develop depression and anxiety during this time, others thrive and have positive outcomes, like reduced risk taking [1]. Why is it that some teens do well during these years, while others may struggle? The differential susceptibility model is a scientific hypothesis that may help explain why children have different ("differential") levels of sensitivity ("susceptibility") to their environments.

\section{WHAT IS DIFFERENTIAL SUSCEPTIBILITY?}

Children who grow up in the same house can turn out to be very different from each other. Why is this? According to the differential susceptibility model, children's natural levels of sensitivity, which stem from normal differences in brain development between children, can cause children growing up in the same environment to have different outcomes, or responses to the same situation. The model suggests there are two types of people: children who are naturally sensitive and children who are naturally resilient, meaning less sensitive. These two groups of children may have differences in how their brains work. It may be hard to tell the difference just by looking at them!

Sensitive children are influenced by their environments. If they are in a negative environment, like a home with lots of fighting, they are more likely have negative outcomes, like depression. But being sensitive is not always bad! If sensitive children are in a positive environment, like a home with lots of love, they do really well. Resilient children are not as sensitive to their environments. This means that, even if they are in negative environments, they are more likely to be ok.

Imagine a turtle and a tortoise. They look very similar on the outside, but their biology makes them very different. Many people get them confused. Turtles and tortoises differ in where they can live. Turtles can survive in the water and on land. Turtles are like the resilient children in the differential susceptibility model, because they have average outcomes in both environments. Tortoises are land animals and cannot swim. In a water environment, they will have terrible outcomes. Tortoises need the land to survive. When they are on land, they have better outcomes compared to turtles. These tortoises are like the sensitive children in the differential susceptibility model (Figure 1).

\section{STUDYING THE BRAIN TO MEASURE DIFFERENTIAL SUSCEPTIBILITY}

Like turtles and tortoises, children are different from each other, even in ways we cannot easily see, such as differences in their 
Figure 1

Differential susceptibility. The hypothesis of differential susceptibility states that while a positive environment is good for everyone, people can have different outcomes in the same negative environment depending on if they are resilient or sensitive in the brain. In this figure, turtles are used to represent resilient individuals and tortoises are used to represent sensitive individuals. The $x$-axis shows the environment type, from water to land. For people, this would be a negative or positive home environment. The $y$-axis shows life outcome, from negative to positive. The resilient turtles (orange) have good outcomes no matter their environment. The susceptible tortoises (green) have outcomes that go from negative to positive, depending on the environment. The tortoises have negative outcomes in the water environment, but they thrive, or have very positive outcomes, if they are in the land environment (adapted from Ellis et al. [2]).

HIPPOCAMPUS

The brain structure that regulates memory and learning.

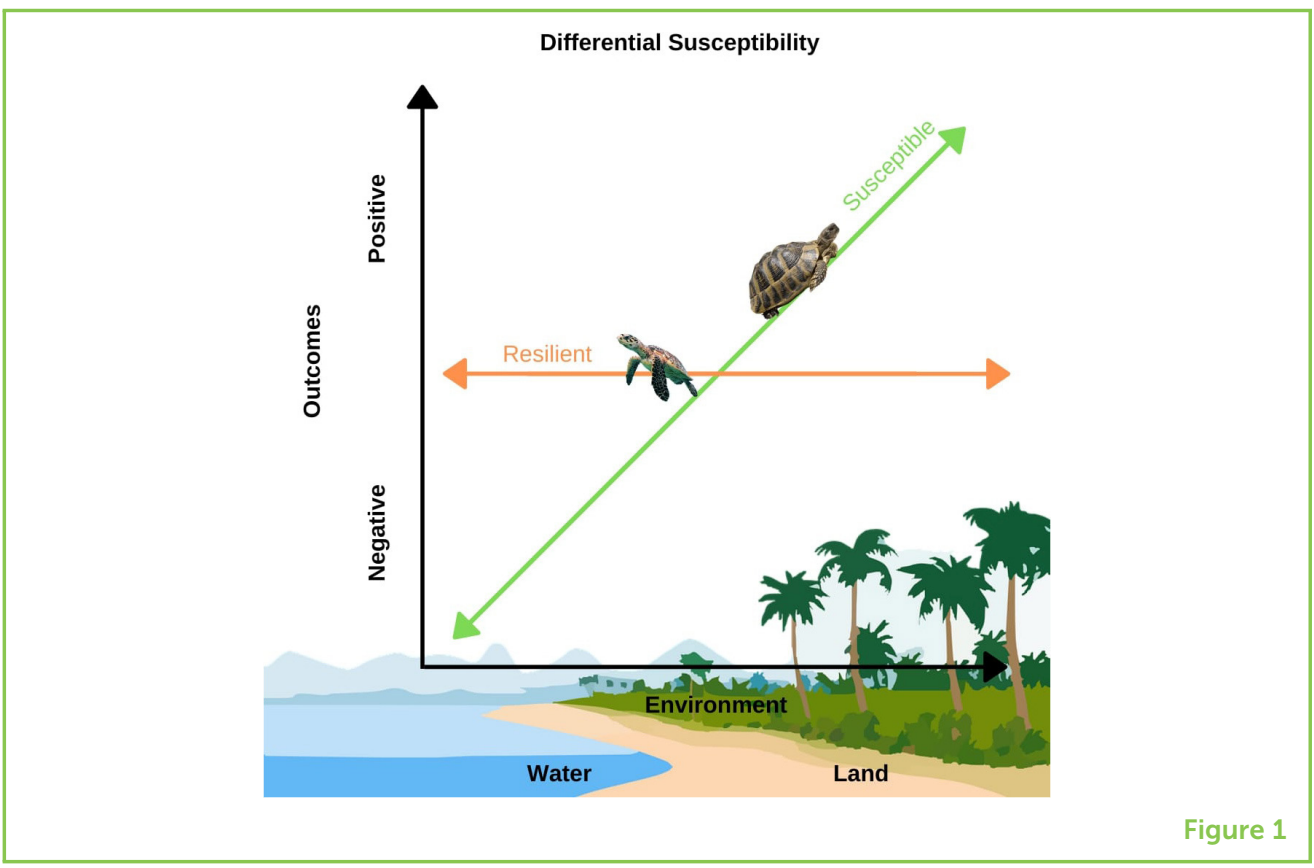

brains. As we get older, the brain changes in its structure (shape and size) and its function (how it works). Scientists can look at changes brain development by using a technology called magnetic resonance imaging (MRI). MRI uses really strong magnets to safely take pictures of the brain [3].

One way that researchers study the brain is to measure the size of different brain regions. To do this, they use a type of MRI called structural MRI (sMRI), which can show researchers how the brain changes in size as children get older. The brain does not just keep getting bigger as we grow up! Some regions actually get smaller, and different parts of the brain grow at different rates.

A second way to study the brain is to measure which areas of the brain are active when we do different things. We use different parts of the brain to do things like reading a book or playing with friends. To measure brain activity, researchers use a different type of MRI called functional MRI (fMRI). fMRI shows researchers how regions of the brain work during different tasks. Examining both brain structure and function allows researchers to identity sensitive and resilient children. Below, we describe two studies that used MRI or fMRI to do this.

\section{DIFFERENTIAL SUSCEPTIBILITY IS RELATED TO THE SIZE OF A CERTAIN BRAIN REGION}

In one study, researchers asked if the size of a brain area called the hippocampus is related to a child's level of sensitivity [4]. The hippocampus is a brain region that helps people build memories and learn from their pasts (Figure 2). The size of the hippocampus 
Figure 2

Brain regions that demonstrate the difference between sensitive and resilient youth. The first image shows the inside of a brain that is facing toward you (front view). The location of the anterior insula (Al) is shown in yellow. The second image shows the inside of a brain when someone is facing to the right (side view). The location of the hippocampus is shown in green, the dorsal anterior cingulate cortex (dACC) is in blue, and the subgenual anterior cingulate cortex (sgACC) is in purple.

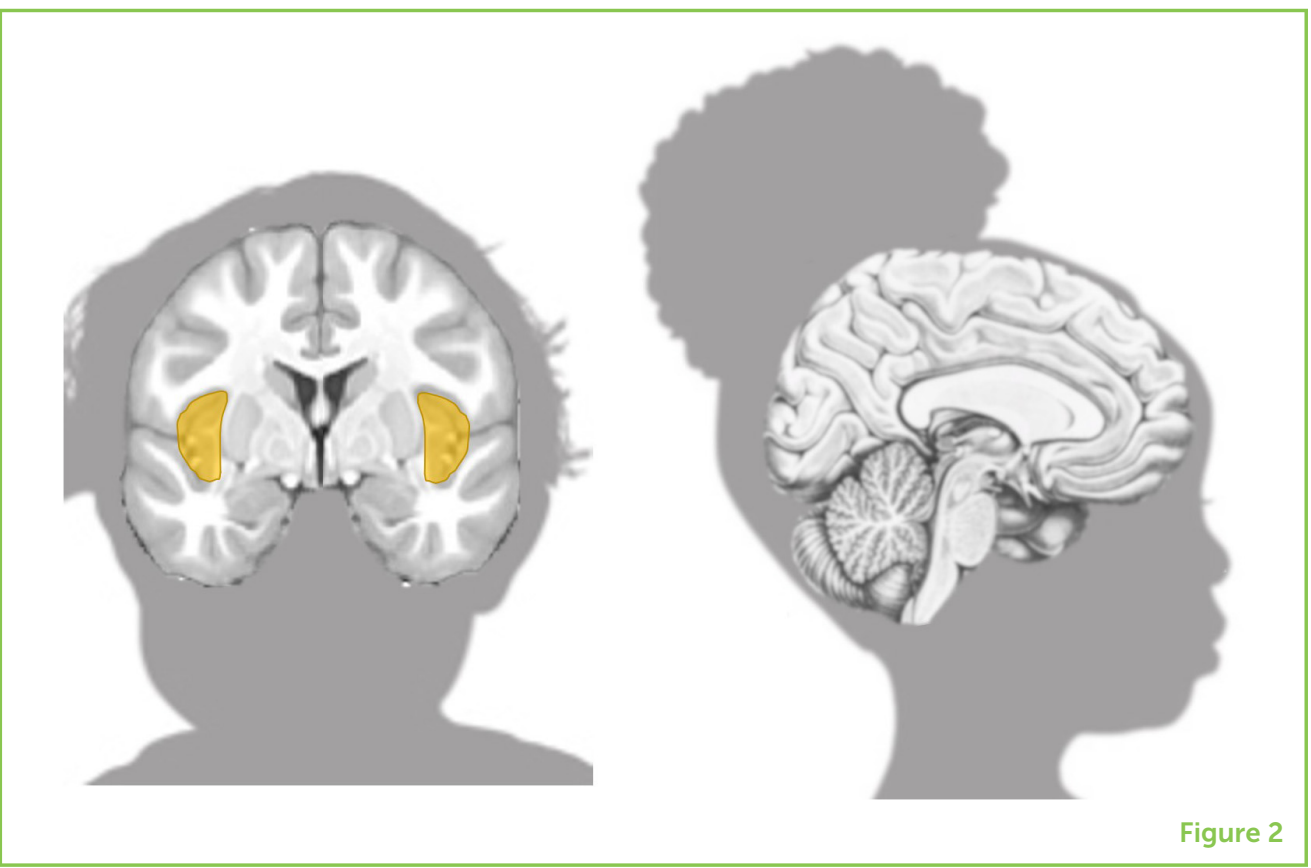

can change when children experience stress while the brain is developing. Using MRI, the researchers measured the size of the teenagers' hippocampi. According to the differential susceptibility model, sensitive adolescents will have larger hippocampi, while resilient adolescents will have smaller hippocampi. The researchers wanted to know if children exposed to maternal aggression have more depression. Maternal aggression is when a mom is mean, yells, or argues with her child. In this study, researchers watched parent-child groups interact to determine the mother's aggression level. In the model, low maternal aggression would be a positive environment, like the land environment for a tortoise. High maternal aggression would be a negative environment, like water for a tortoise. Depression is when someone feels sad and does not want to do anything. Depressed people even feel uninterested in activities that used to make them happy.

The study found that girls with larger hippocampi are more sensitive to maternal aggression. When the sensitive girls experienced high levels of maternal aggression, they had the highest levels of depression. But remember, being sensitive is not always bad! When the sensitive girls experienced low levels of maternal aggression, they thrived, having the lowest levels of depression. Because their outcomes vary based on their environments, they are like a tortoise. In contrast, girls with smaller hippocampi were resilient to maternal aggression. Their depression was low no matter the environment type. These adolescents are like the turtles because they are not sensitive to their environments. 
Figure 3

High dACC brain activation makes youth sensitive to their environment. The purple line shows that the sensitive people, who have high levels of dACC activation, show changing levels of depressive symptoms based on the type of environment they experience. In a stressful environment, they are more likely to have higher depressive symptoms and in a supportive environment, they are less likely to have depressive symptoms. The green line represents resilient people, who have low dACC activation. Their outcomes, in terms of depressive symptoms, are roughly constant no matter which environment they encounter (based on Rudolph et al. [5]).

\section{LIMBIC SYSTEM}

A group of brain structures that are involved with regulating emotions. This system includes the dorsal anterior cingulate cortex (dACC), subgenual anterior cingulate cortex (sgACC), the anterior insula (Al), and other regions.

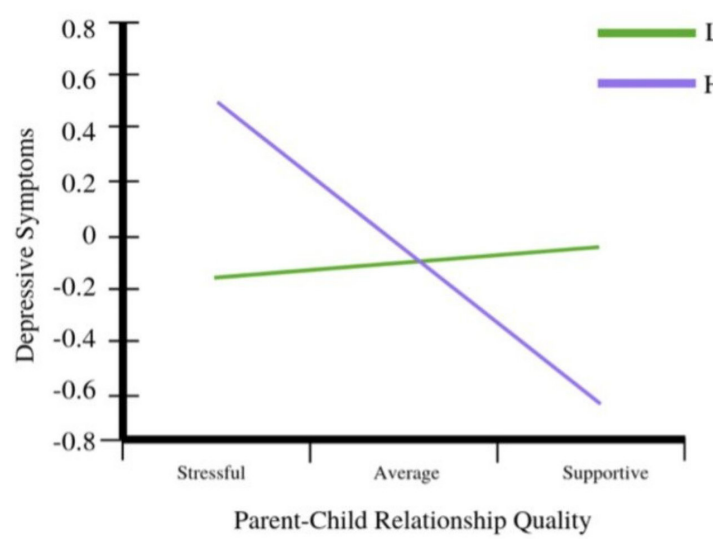

Figure 3

\section{DIFFERENTIAL SUSCEPTIBILITY CAN ALSO RESULT FROM CHANGES IN THE FUNCTION OF CERTAIN BRAIN REGIONS}

Researchers can also measure brain function to look at adolescents' susceptibility to their environments. In another study, researchers used fMRI to measure brain activity in three brain areas of the Limbic System: the dorsal anterior cingulate cortex (dACC), subgenual anterior cingulate cortex (sgACC), and the anterior insula (Al) (Figure 2) [5]. These three regions are part of a group of brain regions that respond to social pain. They are involved in feelings related to social connectedness or exclusion. While they were in the fMRI machine, teens went through a lab task in which they were made to feel socially excluded. According to the differential susceptibility model, children who are really sensitive to feeling excluded will have high activity in these brain areas when they are excluded from their peers during the experiment. Resilient adolescents will show low activity in these brain regions.

The researchers found that girls who showed high brain activity when being excluded in the lab task, meaning that they were more sensitive to feeling excluded, are also more sensitive to their family relationships. In this study, teens answered a survey that asked about their depressive symptoms and how supportive their parents are, for example, if they can talk to their parents about their problems. When the sensitive girls had supportive parent-child relationships, they thrived and showed the lowest depression levels. But when they had stressful parent-child relationships, they suffered and showed the highest depression levels. This means that girls with more activity in the brain regions that respond to social exclusion have higher depressive symptoms when they are in stressful environments, but lower depressive symptoms in supportive environments. They are like the tortoise. Resilient girls showed less brain activity in these same brain regions when they were excluded. They have about the same 
level of depression no matter their home environment. They are like the turtles (Figure 3) [5].

\section{CONCLUSION}

These studies help us to understand why teens can have such different outcomes, even if they grow up in similar environments. Even if we cannot see differences on the outside, studying the brain helps us to see that some teenagers are more sensitive to their environments. Adolescents who are like tortoises and are sensitive show better outcomes in positive environments, but worse outcomes in negative environments. People that are like turtles will have good outcomes no matter what type of environment they experience. However, no matter if you are like a turtle or a tortoise, a positive environment will suit you well!

\section{AUTHOR CONTRIBUTIONS}

MM wrote the first draft of the manuscript. ET revised the manuscript for important intellectual content. Both authors contributed to the manuscript revisions and approved of the submitted version.

\section{REFERENCES}

1. Schriber, R. A., and Guyer, A. E. 2016. Adolescent neurobiological susceptibility to social context. Dev. Cogn. Neurosci. 19:1-18. doi: 10.1016/j.dcn.2015.12.009

2. Ellis, B. J., Boyce, W. T., Belsky, J., Bakermans-Kranenburg, M. J., and Van IJzendoorn, M. H. 2011. Differential susceptibility to the environment: an evolutionary-neurodevelopmental theory. Dev. Psychopathol. 23:7-28. doi: 10.1017/S0954579410000611

3. Hoyos, P., Kim, N., and Kastner, S. 2019. How is magnetic resonance imaging used to learn about the brain? Front. Young Minds 7:86. doi: 10.3389/frym.2019. 00086

4. Whittle, S., Yap, M. B., Sheeber, L., Dudgeon, P., Yucel, M., Pantelis, C., et al. 2011. Hippocampal volume and sensitivity to maternal aggressive behavior: a prospective study of adolescent depressive symptoms. Dev. Psychopathol. 23:115-29. doi: 10.1017/S0954579410000684

5. Rudolph, K. D., Davis, M. M., Modi, H., Fowler, C., Kim, Y., Miernicki, M. E., et al. 2018. Differential susceptibility to parenting in adolescent girls: moderation by neural sensitivity to social cues. J. Res. Adolesc. 30:177-91. doi: 10.1111/jora. 12458

SUBMITTED: 16 October 2019; ACCEPTED: 23 July 2020;

PUBLISHED ONLINE: 08 September 2020.

EDITED BY: Marcel Ruiz-Mejias, Pompeu Fabra University, Spain 


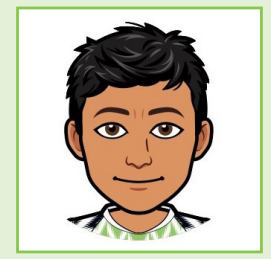

CITATION: McBride M and Telzer EH (2020) Why Are Some Kids More Sensitive to Their Environments? Front. Young Minds 8:113. doi: 10.3389/frym.2020.00113

CONFLICT OF INTEREST: The authors declare that the research was conducted in the absence of any commercial or financial relationships that could be construed as a potential conflict of interest.

COPYRIGHT ( 2020 McBride and Telzer. This is an open-access article distributed under the terms of the Creative Commons Attribution License (CC BY). The use, distribution or reproduction in other forums is permitted, provided the original author(s) and the copyright owner(s) are credited and that the original publication in this journal is cited, in accordance with accepted academic practice. No use, distribution or reproduction is permitted which does not comply with these terms.

\section{YOUNG REVIEWER}

\section{LUCAS, AGE: 15}

My name is Lucas, I am a tenth grader at the Brookline High School. I speak English, Spanish, and I am currently taking Chinese and I have been learning it since second grade. I like playing and watching soccer and also playing Xbox.

\section{AUTHORS}

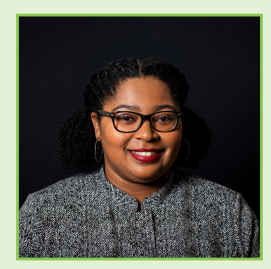

\section{MARGARETT MCBRIDE}

I am a graduate student at The University of North Carolina at Chapel Hill. I study how neighborhood experiences impact Black and Latinx families. I am currently researching how positive factors in neighborhoods and families (e.g., sense of belonging, community programs, racial socialization, involved parenting) can help teens positively cope through their experiences with violent neighborhoods. In my spare time, I like making art, going to art museums, and volunteering at community festivals. ${ }^{*}$ marsmalive.unc.edu

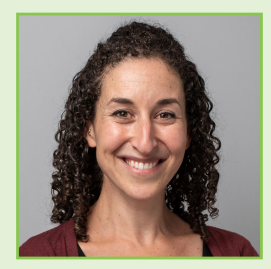

\section{EVA TELZER}

I am an Associate Professor at The University of North Carolina at Chapel Hill. I study how teenagers relate to their peers and families, how their brains develop, and how this impacts their well-being like feelings of depression, risk taking, or prosocial behaviors like helping and empathy. The teenage years are sometimes difficult, but I try to understand how teens can thrive and how this time can set the stage for a lifetime of flourishing. In my free time, I like drawing, hiking with my dog, and reading a good book. 